\title{
Sinonasal T Cell Lymphoma: A Case Report
}

\author{
Deviprasad Shetty - Rajeshwari Aroor • \\ K. S. Gangadhara Somayaji - Mohammed Tahir • \\ N. A. Mohammad
}

Received: 9 March 2008/Accepted: 15 August 2008/Published online: 10 April 2011

(C) Association of Otolaryngologists of India 2011

\begin{abstract}
Sinonasal lymphomas are aggressive locally destructive midfacial necrotizing lesions. Most of them initially diagnosed as lethal midline granulomas, a term which is slowly replaced by sinonasal lymphoma. Here is one such case report of sinonasal $\mathrm{T}$ cell lymphoma where there was a difficulty in diagnosis and required an incisional biopsy.
\end{abstract}

Keywords Sinonasal T cell lymphoma . Incisional biopsy · Immunohistochemistry

\section{Introduction}

Sinonasal lymphomas are relatively rare. Malignant lymphomas are associated with widespread necrosis, inflammation and surface crusting, which often resembles granulomatous diseases of the nose. They were earlier considered as lethal midline granulomas [1]. Thanks to the introduction of immunohistochemistry, now, most of these tumours are better understood and better managed.

\section{Case History}

A 55 year old man, a known diabetic and hypertensive patient presented with bilateral nasal obstruction of 2 years duration with intermittent epistaxis of 6 months duration. He also gave the history undergoing a septoplasty followed by biopsy from a nodular lesion inside the nose about

D. Shetty · R. Aroor · K. S. G. Somayaji $(\bowtie) \cdot$ M. Tahir .

N. A. Mohammad

Yenepoya Medical College, Mangalore, India

e-mail: ksgsomayaji@gmail.com
10 months back. The biopsy was inconclusive showing only inflammatory cells and his symptoms did not improve and the patient was referred to us. On examination, patient had a swelling over the nasal dorsum with skin discoloration and induration with multiple small nodular lesions in both the nasal vestibule (Fig. 1). On anterior rhinoscopy, there was crusting and inflamed nasal mucosa with reduced nasal airway on both sides. Small nodular lesions were also seen on the nasal septum and inferior turbinate anteriorly similar to the lesions seen in the vestibule. Posterior rhinoscopy revealed only crusting. Diagnostic nasal endoscopy was showing inflamed nasal mucosa and the scope could not be advanced beyond the middle turbinate on both the sides because of narrowed nasal passage. CT scan showed a soft tissue density mass in the lateral nasal wall with thickening of nasal mucosa without involvement of paranasal sinuses (Fig. 2). Initial biopsy from the nodular lesions was again inconclusive showing inflammatory cells. The patient was therefore posted for incisional biopsy under GA. Multiple biopsies were taken from the vestibule on both sides and lateral nasal wall through intercartilaginous incision. Histopathological report was suggestive of T cell lymphoma (Fig. 3). The diagnosis was further confirmed by immunohistochemistry which was positive for CD 45 and negative for CD3 and CD20 (Fig. 4). Patient was treated with multidrug chemotherapy followed by radiotherapy, a total dose of 40 Gy over a period of 4 weeks. The patient responded well to the treatment.

\section{Discussion}

In the past sinonasal lymphomas were confused with various infections, autoimmune and inflammatory diseases. Now, they are considered to represent peripheral $\mathrm{T}$ cell 


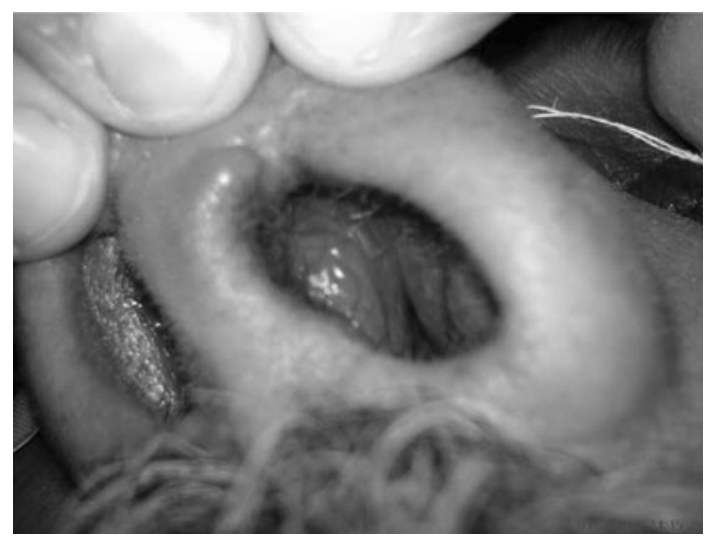

Fig. 1 Nodules in the vestibule

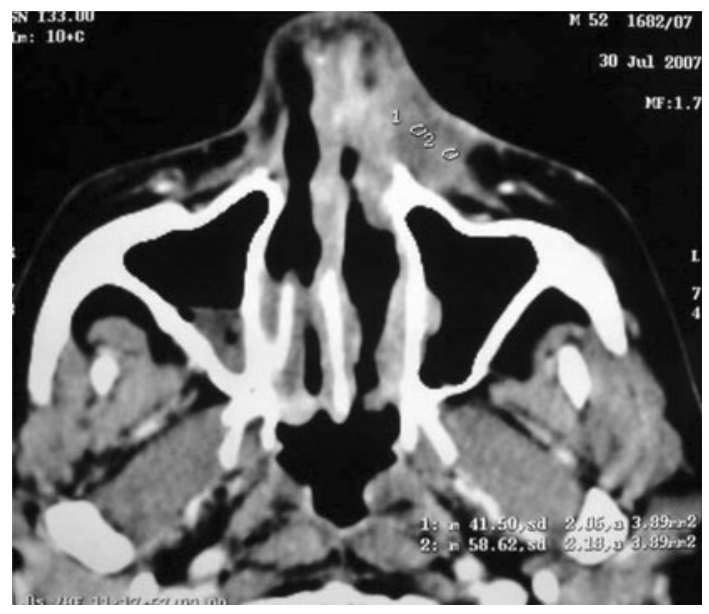

Fig. 2 Thickening of lateral nasal wall

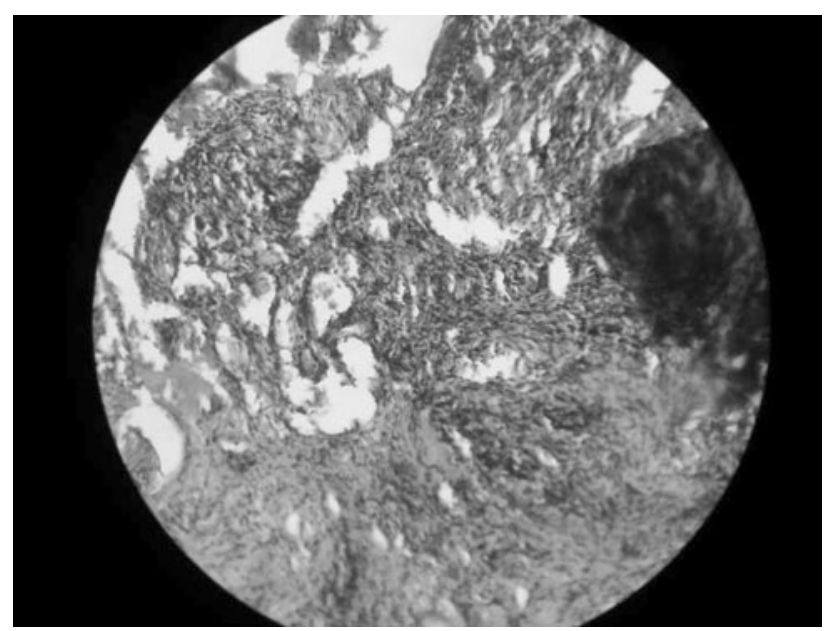

Fig. 3 HPE Photograph

lymphomas. In Asian population, more than $90 \%$ of sinonasal lymphomas are of $\mathrm{T}$ cell origin [2] and most of them are NK cell in origin [2]. Nasal T cell lymphomas usually originate from the nasal cavity and invade the adjacent structures where as the sinonasal B cell lymphomas usually arise from the paranasal sinuses and is more common in the western population [2]. The usual presenting symptoms of sinonasal lymphomas are nasal obstruction, discharge, epistaxis, unilateral facial and cheek swelling, headache and symptoms secondary to tumour extension [2].

The diagnosis of destructive diseases of the sinonasal region depends on clinical and pathological findings as imaging in these lesions may not reveal definite diagnosis. Nasal obstruction and nasal discharge which are commonly seen in sinonasal lymphoma are also seen in rhino sinusitis as well [3].

The diagnosis of sinonasal lymphoma is difficult because of the presence of ischemic type of necrosis, interspersed inflammatory cells, polymorphous composition of neoplastic cells. The main differential diagnosis for sinonasal lymphoma is Wegener's granulomatosis. At times, it may be difficult to distinguish these diseases histilogically [4]. Adequate amount of biopsy material and immunohistochemistry helps in such situations. Sinonasal lymphoma cases express $\mathrm{T}$ cell markers such as CD 2, CD 45RO and CD 43 and often express CD 56. CD 20 is universal B cell marker. The diagnosis of Wegener's granulomatosis is based on histopathological features like the presence of non caseating multinucleated giant cell granulomas and necrotizing vasculitis. The diagnosis is aided by elevated titers of anti neutrophil cytoplasmic antibodies [3, 4].

Because of the presence of multiple nodular lesions in the nasal vestibule and the crusting in the nasal cavity, this case was initially thought to be rhinoscleroma. Histopathology is confirmatory in rhinoscleroma which shows Russell bodies and Mikulicz cells. In addition, all other chronic inflammatory and granulomatous diseases of the nose will have to be kept in mind as differential diagnosis. Also, trauma and cocaine abuse can be considered as differential diagnosis as these entities are important causes of septal perforation which is a common feature in sinonasal lymphoma [3], though not seen in our case.

Nasal $\mathrm{T}$ cell lymphomas respond well to multidrug chemotherapy followed by field radiotherapy [1, 5]. However, Kyubo Kim et al. [6] in their study, feel that chemotherapy should be tried after the local control is attained with radiotherapy. In spite of this; death from this disease occurs in 50\% of patients as a result of distant extra nodal spread or relapse [1,5]. Patients with sinonasal lymphomas have a better prognosis compared to those with nodal lymphomas of similar grades.

\section{Conclusion}

Diagnosis of sinonasal lymphoma is often difficult. The purpose of publication of this article is for its rarity, the 


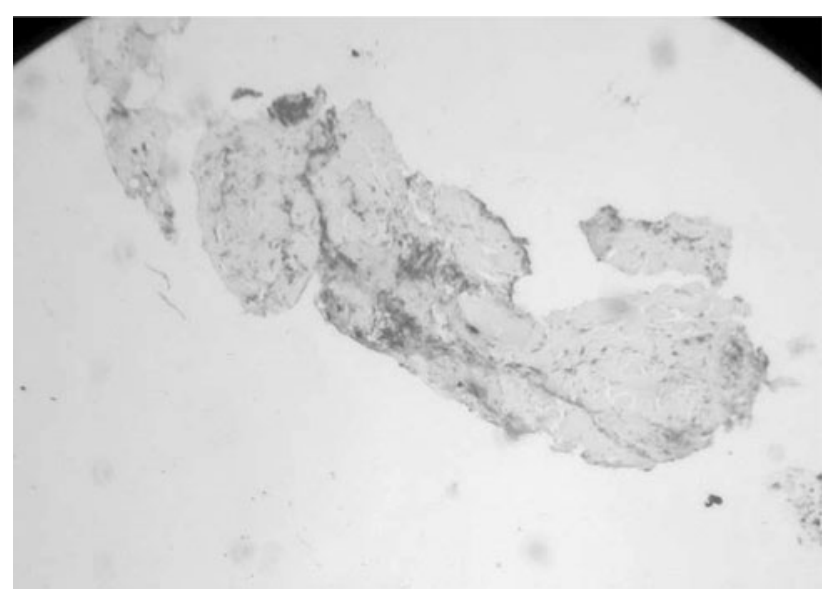

Fig. 4 Immunohistochemistry slide positive for CD45

difficulty in diagnosis, the need for adequate biopsy material which may require an incisional biopsy and the need for immunohistochemistry. A careful examination and adequate biopsy avoids an unwarranted delay in the diagnosis and also unnecessary procedures like septoplasty as was done in our case previously.

\section{References}

1. Patel V, Mahajan S, Kharkar V, Khopkar U (2006) Nasal extra nodal NK/T-cell lymphoma presenting as a perforating palatal ulcer. Indian J Dermatol Venereol Leprol 72(3):218-221

2. Kamath MP, Kamath G, Bhojwani K, Pai M, Shameem A, Agarwal S (2006) Sininasal lymphoma: a case report. Ear Nose Throat J 85(5):325-327

3. Borges A, Fink J, Villablanca P, Eversole R, Lufkin R (2000) Midline destructive lesions of the sinonasal tract: simplified terminology based on histopathologic criteria. AJNR 21(2):331-336

4. Abbondanzo SL, Wenig BM (1995) Non Hodgkin's lymphoma of the sinonasal tract. Cancer 75(6):1281-1290

5. Gourin CG, Johnson JT, Selvaggi K (2001) Nasal T cell lymphoma: case report and review of diagnostic features. Ear Nose Throat J 80:458-460

6. Kim K, Eui KC, Chul WK II, Han K, Park C II (2005) Treatment outcome of angiocentric T cell and NK cell lymphoma, nasal type: radiotherapy versus chemoradiotherapy. Jpn J Clin Oncol 35(1): $1-5$ 